\title{
Final Report for DoE Grant DE-FG02-03ER54712,
}

\section{Experimental Studies of Collisionless Reconnection Processes in Plasmas}

\author{
Miklos Porkolab, PI
}

Jan Egedal, Co-PI

\begin{abstract}
MIT Physics Department and Plasma Science and Fusion Center Cambridge, MA, 02139
\end{abstract}

\section{Executive summary}

The Grant DE-FG-02-00ER54712, "Experimental Studies of Collisionless Reconnection Processes in Plasmas", financed within the DoE/NSF, spanned a period from September, 2003 to August, 2007. It partly supported an MIT Research scientist, two graduate students and material expenses. The grant enabled the operation of a basic plasma physics experiment (on magnetic reconnection) at the MIT Plasma Science and Fusion Center and the MIT Physics Department. A strong educational component characterized this work throughout, with the participation of a large number of graduate and undergraduate students and interns to the experimental activities.

The study of the collisionless magnetic reconnection constituted the primary work carried out under this grant. The investigations utilized two magnetic configurations with distinct boundary conditions. Both configurations were based upon the Versatile Toroidal Facility (VTF). The first configuration is characterized by open boundary conditions where the magnetic field lines interface directly with the vacuum vessel walls. The reconnection dynamics for this configuration has been methodically characterized and it has been shown that kinetic effects related to trapped electron trajectories are responsible for the high rates of reconnection observed [7]. This type of reconnection has not been investigated before. Nevertheless, the results are directly relevant to observations by the Wind spacecraft of fast reconnection deep in the Earth magnetotail [9].

The second configuration was developed to be specifically relevant to numerical simulations of magnetic reconnection, allowing the magnetic field-lines to be contained inside the device. The configuration is compatible with the presence of large current sheets in the reconnection region and reconnection is observed in fast powerful bursts. These reconnection events facilitate the first experimental investigations of the physics governing the spontaneous onset of fast reconnection [12].

In this Report we review the general motivation of this work, the experimental set-up, and the main physics results. The details of the individual chapters are naturally contained in the relevant publications [1-12], indicated in the reference list and annexed to this Report. 


\section{The problem of magnetic reconnection (from original proposal)}

Magnetic reconnection [13] plays a fundamental role in magnetized plasmas as it permits rapid release of magnetic stress and energy through changes in the magnetic field line topology. It controls the spatial and temporal evolution of explosive events such as solar flares, coronal mass ejections, and magnetic storms in the Earth's magnetotail driving the auroral phenomena [14-17]. Because of its unique importance to understanding violent macroscopic phenomena in nearly all magnetized plasmas, reconnection has been the subject of an increasing number of theoretical investigations. Despite the intense research over the past decades, the origin of the fast time scales under which collisionless magnetic reconnection is observed, is still poorly understood [18]. In recent years it has been shown that so-called two fluid effects (related to the large difference between the electron mass and the ion mass) play an important role in determining the rate of reconnection. However, a detailed understanding of the electron dynamics is still needed to explain the spontaneous onset of reconnection, characteristic for reconnection on the surface of the sun, in the Earth's magnetotail, and in magnetic fusion devices.
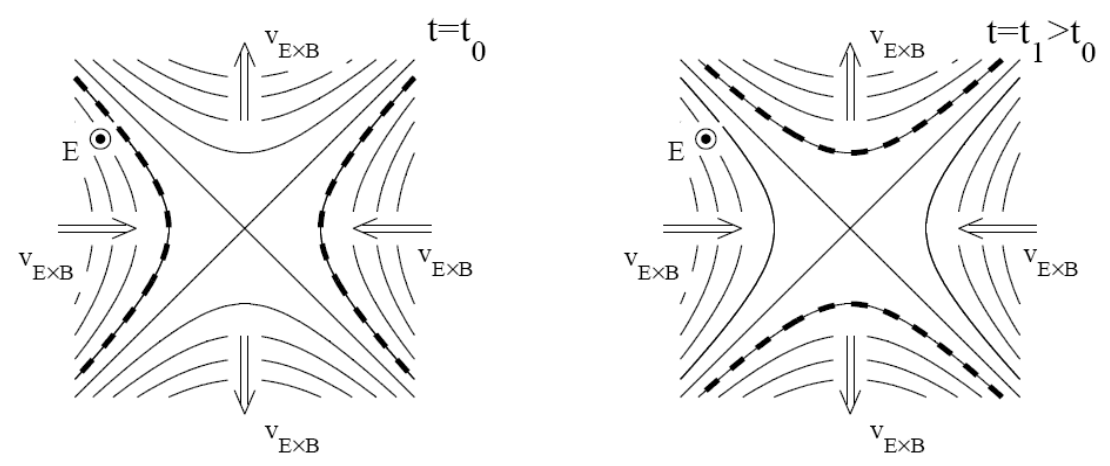

Figure 1: Illustration of the basic geometry for fast magnetic reconnection.

During magnetic reconnection, magnetic field lines in opposing directions cross link and form a magnetic cusp. Such a configuration is characterized by an X-line, a line along which the two components of the magnetic field perpendicular to the line vanish. This basic geometry for fast magnetic reconnection is given in Fig. 1. The sharp gradients and the fine structure in the Xline geometry is thought to cause the breakdown of the theory of resistivemagnetohydrodynamics, which predict, in contradiction to observations, that reconnection is slow in collisionless plasma.

In order to make the reconnection problem numerically and analytically tractable, theoretical investigations often consider a fluid formulation of the plasma dynamics. The mostused theoretical framework for the study of reconnection is the two-fluid plasma description from which the generalized Ohm's law follows

$$
\mathbf{E}+\mathbf{v} \times \mathbf{B}=\eta \mathbf{j}+\frac{1}{n e}\left(\mathbf{j} \times \mathbf{B}-\nabla \cdot \mathbf{p}_{e}\right)+\frac{m_{e}}{n e^{2}}\left(\mathbf{v} \cdot \nabla \mathbf{j}+\frac{\partial \mathbf{j}}{\partial t}\right)
$$

Theoretical models for reconnection have been developed in which one or more of the terms on the right hand side of equation (1) are elected to be responsible for breaking the socalled ideal plasma "frozen-in law." As an example, the well-known Sweet-Parker reconnection model $[19,20]$ employs only the first term $(\eta j)$; this yields reconnection via resistive diffusion through an elongated current sheet. In space and laboratory plasmas, in which the collision rate is small, the Sweet-Parker model is unable to explain the fast rates of reconnection observed; 
however, due to its simplicity, it remains a useful reference scenario for experimental and theoretical investigations.

In other theoretical work, fast reconnection was accounted for by introducing an anomalously high resistivity, $\eta^{*}$, which speeds the rate of magnetic field diffusion. It was argued that the anomalous resistivity stems from instabilities that are produced by the relative streaming of electrons and ions in current layers. The scattering of the electrons in the associated electric field would enhance the drag on the electrons and facilitate an anomalously high rate of momentum transfer between the ions and electrons [21]. For experimental studies, detailed theoretical predictions are available which describe the level and type of turbulence needed to explain fast reconnection [22, 23].

In recent years, the effect of the Hall-term, $\mathbf{j} \times \mathbf{B} /(n e)$, and the related dispersive waves have been studied intensely [23]. In the absence of a magnetic guide field (a magnetic field along the $\mathrm{x}$ - line), these analytical and numerical studies indicate that the Hall-term may play a key role in fast reconnection. The ions are thought to be unmagnetized at length scales of the ion skin depth $c / \omega_{p i}$, while the electrons are magnetized down to the electron skin depth, $c / \omega_{p e}$. At length scales smaller than $c / \omega_{p i}$, the Hall term is important through the presence of characteristic inplane currents. Due to the dispersion relation of the associated dispersive waves, the reconnection rate becomes insensitive to the mechanism that is responsible for breaking the "frozen-in law" [24]. In the presence of a guide magnetic field, not only the Hall term, but also effects related to the electron pressure tensor $\mathbf{p}_{\mathrm{e}}$, are expected to be important $[25,26]$. In most reconnection models, the ion sound Larmor radius, $\rho_{s}=\left(m_{i} T_{e}\right)^{1 / 2} / q B$, becomes the characteristic length scale for pressure perturbations in the X-line region. The associated asymmetry in the density profile has been related to the physics of the kinetic Alfvén waves [27] and energization of electrons has been documented numerically in the density depleted parts of the separatrix [28, 29].

In general, the pressure tensor is anisotropic and includes off-diagonal elements, which account for a finite plasma viscosity. However, nearly all analytical work on magnetic reconnection relies on the simplifying assumption that the pressure is isotropic so that the pressure terms can be described by a scalar function. This is also the case for most numerical fluid simulations, although examples exist which include anisotropic pressures components $\left(\mathrm{p}_{\|}\right.$, $\left.\mathrm{p}_{\perp}\right)$, parallel and perpendicular to the magnetic field.

The electron inertia term, $\left(m e /\left(n e^{2}\right)\right)(d \mathbf{j} / d t)$, is important in time dependent models; the convective component, $v \cdot \nabla$, can also be important in steady state $[30,31]$. This is the case when the characteristic length scale for the current profile is comparable to the electron skin depth, $c / \omega_{p e}$. In many models this term is crucial in obtaining electron momentum balance at the $\mathrm{x}$-line.

Fluid formulations rely on the ansatz that the particles cannot sample macroscopic length scales in their motion. This is best achieved when the particles are well magnetized, so that their motion perpendicular to the magnetic field is constrained, and when their parallel mean free path is short. This assumption is fundamental in deriving the fluid models from the kinetic equations and may not be fulfilled during collisionless reconnection. On the other hand, with the development of computational tools and resources, the number of investigations that use particle simulations has increased dramatically. Particle codes simulate the plasma from first principles and are, therefore, likely to capture the important physics for reconnection. Potentially, the boundary conditions applied will impact the results of the simulations, so an important detail of such simulations is to specify realistic boundary conditions. Periodic boundary conditions are the most popular choice owing to their numerical stability and simplicity, but in recent years, codes have been developed that employ so-called "open" boundaries [32].

The appropriateness of the various boundaries remains a subject of controversy. It is likely that the controversy may only be settled through direct observation of reconnection in space or laboratory plasmas. These facts call for caution when applying the fluid models and particle codes to the reconnection problem and call for a careful comparison of theoretical and code predictions with detailed experimental observations in the collisionless domain. 


\section{Reconnection experiments (from original proposal)}

A number of experiments have been specifically designed in the past to address some of the issues related to the plasma behavior around the magnetic X-point and to study the magnetic reconnection in general [33-40]. Two parameters important for characterizing the experiments are the ratio of the ion Larmor radius to the plasma size $\left(\rho_{i} / L\right)$ and the magnetic Lundquist number $S=\mu_{0} L v_{A} / \eta$, where $v_{A}$ is the Alfvén speed. A large value of $\mathrm{S}$ is required such that the magnetic fields do not to diffuse through the plasma on time scales shorter than the Alfvén time.

Some of the early experiments were based on a linear plasma geometries, with $S>1$, but with $\rho_{i} / L>1$ (i.e. un-magnetized ions) [33-35]. In these configurations, the effective plasma resistivity, $\eta^{*}=E / j$, was observed to be one to two orders of magnitude larger than its classical value. Under these conditions, the anomalous resistivity was found to be caused mainly by turbulence and instabilities. In addition to un-magnetized ions, linear devices are characterized by end effects that introduce additional complications in the interpretation of reconnection results: The electrostatic sheaths at the two ends make determination of the electric field in the body of the plasma difficult due to substantial potential drops in the sheaths.

To overcome this limitation, more recent experiments employ the technique of merging two spheromak plasma tori to induce reconnection. These experiments are characterized by "MHD-like" plasmas, $\rho_{i} / L<<1$, large Lundquist numbers $(S>100)$, and relatively high collisionality $\left(\lambda_{e} / L<1\right.$, where $\lambda_{e}$ is the electron mean free path). The formation and the evolution of the current layer in the magnetic X-point region is accompanied by the reconstruction of the magnetic flux lines, in space and time, using arrays of internal magnetic probes. The 3D character of magnetic reconnection, including the role of a guide magnetic field in co- and counter-helicity plasma merging, was investigated in the TS-3 experiment [38]. Ion heating and acceleration was observed via Doppler shifts and broadening of plasma spectral lines in [41]. A correlation between magnetic reconnection and the generation of energetic ion flows was establish [42]. In the SSX experiment, two spheromak plasmas interact through cutouts in a wall [40]. This interaction is intrinsically three dimensional, and it reveals an out of plane magnetic field generated by the plasma during the reconnection process [43].

The MRX experiment [39] was also designed to study the collision of two spheromak plasmas. However, so far reconnection has been driven by inducing an electric field in the region of a magnetic null between two internal coils. The plasma is formed by an inductive discharge, and the electric field driving the reconnection is generated by varying the poloidal flux over a short time scale $(<50 \mu \mathrm{s})$. The experimental investigations indicated that a generalized version of the Sweet-Parker model, including an enhanced resistivity, compressibility, and downstream plasma pressure, could explain the observed geometry of the current sheets. The effective resistivity, measured to be up to a factor of 10 larger than the Spitzer resistivity, enhances as the collisionless regime is approached, i.e. when the mean free path exceeds the thickness of the current sheet. The origin of this enhancement is still not clear; lower-hybrid-drift turbulence has been detected but did not correlate with the enhancement in the resistivity [44].

The VTF (Versatile Toroidal Facility) magnetic reconnection experiment was developed at the Plasma Science and Fusion Center [1]. The experiment was designed to investigate the detailed structure and dynamics of the diffusion region in magnetic reconnection. The focus of the experiment is on plasma flows, particle orbits and dynamics, the evolution of the magnetic and electric field structures, and the development of instabilities during the reconnection process. 


\section{The VTF open cusp experiment}

The first experimental campaign was completed in an open cusp configuration where the magnetic field lines interface with the wall of the vacuum vessel. New significant results are still anticipated in this configuration where collisionless reconnection is routinely achieved at rates that are four orders of magnitude above the classical predictions. The VTF experiment is the first in which a significantly enhanced reconnection rate has been observed in the presence of a guide magnetic field. It is also the only dedicated reconnection experiment operating in the collisionless regime, $\lambda_{e} / L>100$, for which the ions are magnetized and for which the guide magnetic field can be varied continuously.
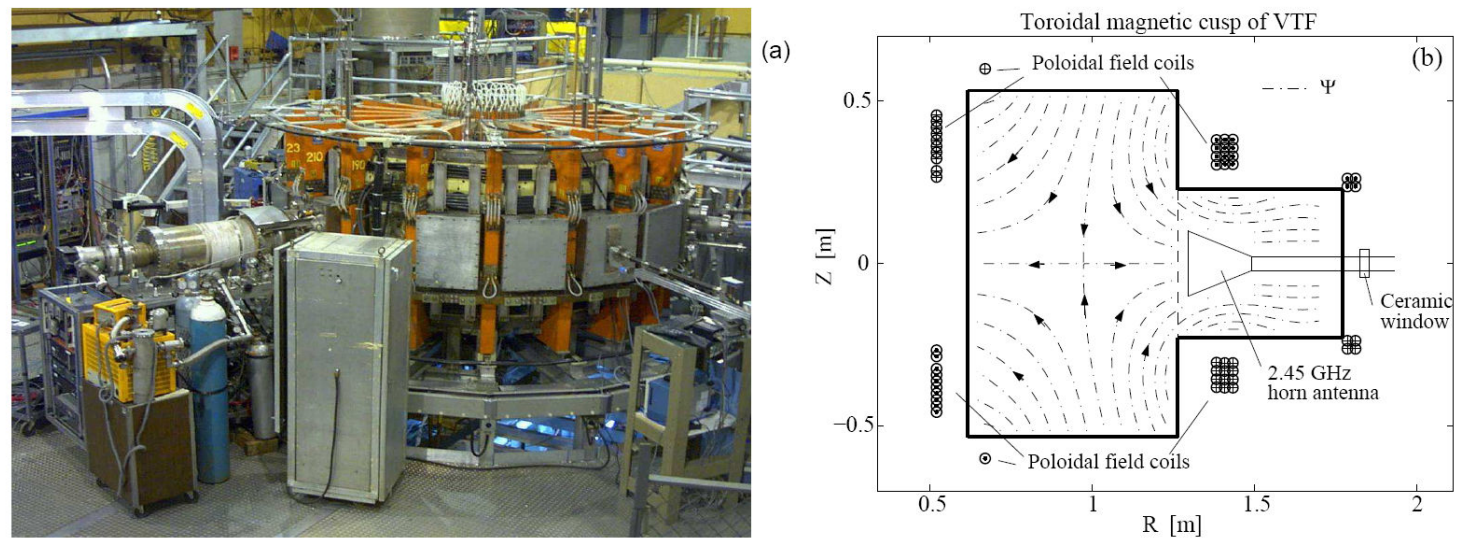

Figure 2: (a) Picture of the VTF device. (b): Poloidal cross section of VTF. The solid contour lines represent the poloidal magnetic field magnitude. The dashed contour lines correspond to constant levels of the poloidal magnetic flux, $\Psi$, which coincide with magnetic field lines.

The plasmas are produced by electron cyclotron resonance heating (ECRH) injecting 20 $\mathrm{kW}$ rf power at $2.45 \mathrm{GHz}$. Typical plasma density and electron temperature are in the range of $n$ $\approx 10^{17} \mathrm{~m}^{-3}$ and $T e \approx 15 \mathrm{eV}$. Due to the relatively large aspect ratio of the VTF device, the magnetic geometry in the vicinity of the $X$ line is well approximated by the following expression: $\mathbf{B}=b_{0}\left[\left(R-R_{0}\right) \hat{\mathbf{R}}+Z \hat{\mathbf{Z}}+l_{0} \hat{\boldsymbol{\phi}}\right]$,

The toroidal magnetic cusp configuration of the VTF is applied for the study of magnetic reconnection in the collisionless regime. The device and its poloidal cross section is illustrated in Fig. 2. The poloidal magnetic field, $\mathbf{B}_{\text {cusp }}$, is described by an $X$-configuration with an absolute magnetic null for $(R, Z)=(0.98,0) \mathrm{m}$. The strength of the poloidal field is characterized by $\mid$ grad $B_{\text {cusp }} \mid$. In addition to the poloidal magnetic field, a toroidal guide magnetic field, $B_{g}$, may be applied. The ratio between the cusp field and the guide field is given by $r=l_{0}$, where $l_{0}=$ $B_{g} /\left|\nabla B_{\text {cusp }}\right|$ and $r$ is the distance from the $X$-line. The reconnection drive is produced by the transformer action of a solenoid installed at the center of the device. The maximum driven reconnection rate is $E_{\varphi} \approx 10 \mathrm{~V} / \mathrm{m}$, which is maintained in nearly steady-state up to $1 \mathrm{~ms}$. The profiles of the number density, current density, and electrostatic potential are obtained by movable electrostatic and magnetic probe arrays [2]. Data are collected during a large number of discharges, taking advantage of the high degree of shot-to-shot reproducibility. 


\subsection{Driven reconnection}

The data in Fig. 3 represents the measured time evolution of these profiles for a hydrogen plasma for $B_{g} \approx 0.087 \mathrm{~T}$ and $l_{0}=7 \mathrm{~m}$. The data corresponds to the initial phase after the reconnection drive is switched on at $t_{0}$. The profiles of the three components of $\mathbf{B}$ are obtained from magnetic probe measurements. The plasma currents are calculated through $\mu_{0} \mathbf{j}=\nabla \times \mathbf{B}$, assuming toroidal symmetry. The dashed lines in all the subfigures represent magnetic field lines, inferred on the basis of the toroidal plasma current profile and the currents applied in the VTF coil sets [2]. Two magnetic field lines, corresponding to one particular value of the poloidal magnetic flux function $\Psi$, are highlighted in pink so that they can be followed in time as they drift together, reconnect, and drift apart.

The first row of profiles represents the evolution of the plasma density. Because of the nature of the plasma production, a region of increased density is observed for $R>1 \mathrm{~m}, Z<0 \mathrm{~m}$, and $t=0 \mu \mathrm{s}$. As time progresses, this area of increased density can be followed as it propagates along with the magnetic field lines, which move in the poloidal cross section and undergo reconnection. The inflow and outflow velocity is given by the $E \times B$ velocity imposed by the external reconnection drive.

The second row shows the temporal evolution of the current profile. For $t=12 \mu \mathrm{s}$ and $t=$ $24 \mu \mathrm{s}$, the data reveals a characteristic diamond shaped profile. The current density does not exceed $3 \mathrm{kA} / \mathrm{m}^{2}$. This value is about 3 orders of magnitude lower than the value $E / \eta_{s}$, where $\eta_{s}$ is the Spitzer resistivity, calculated using the measured electron temperature. The third row in Fig. 3 shows the evolution of the electrostatic potential, $\Phi$. The potential evolves towards a configuration where $E \cdot B=0$ away from the $X$-line [3]. With the knowledge of the electrostatic potential, $\Phi$, it is possible to infer the total electric field $\mathbf{E}=-\nabla \Phi-\partial \mathbf{A} / \partial t$; hence the size of the diffusion region where $E \cdot B \neq 0$ (here $\mathbf{A}$ is the magnetic vector potential).

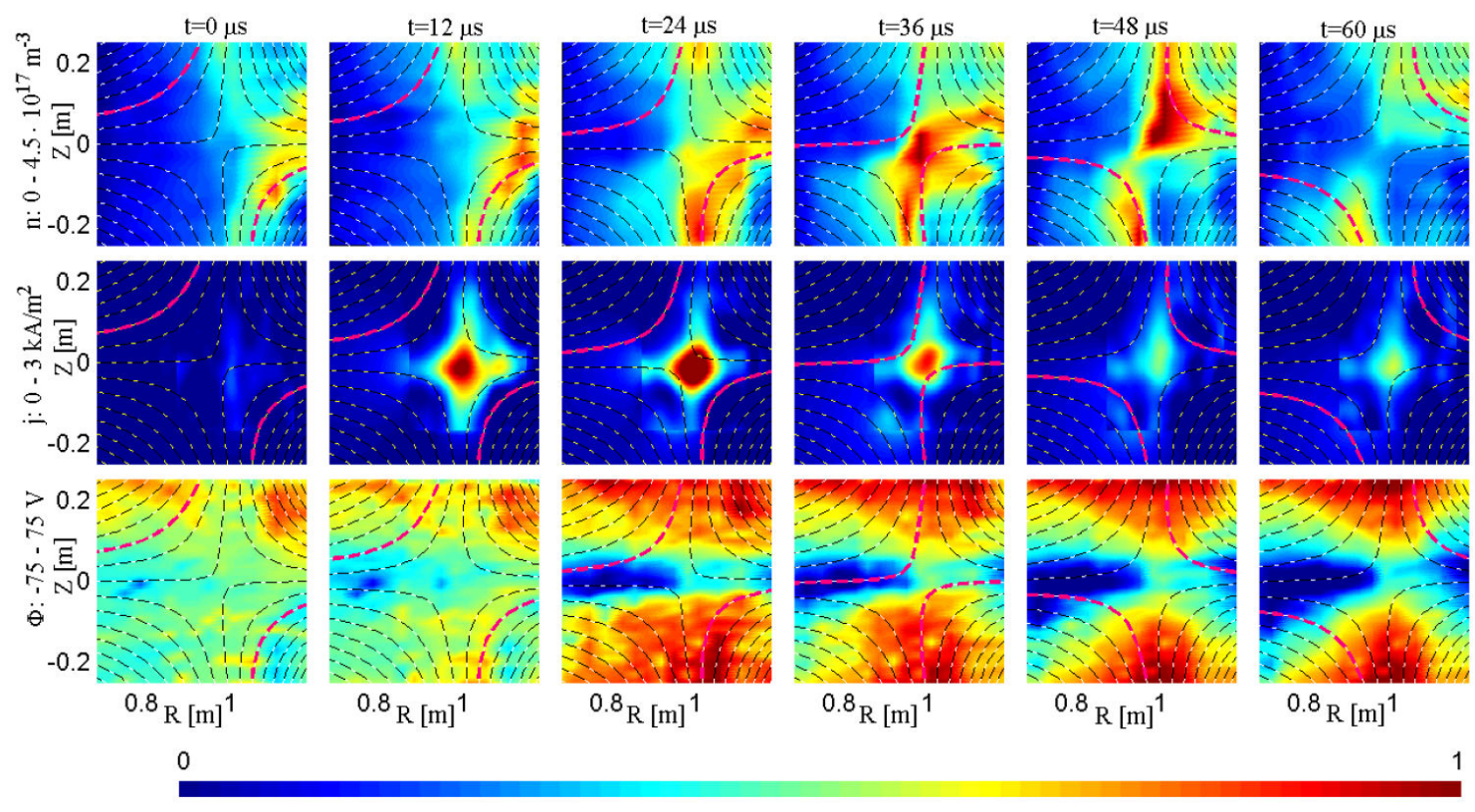

Figure 3: Measured contours of density, plasma current, and electrostatic potential during the initial phase of the forced reconnection pulse. Each row of subfigures corresponds to a snapshot in time as indicated in the left hand side. Between each row, the time is incremented by $12 \mu \mathrm{s}$. 
In Figs. 4(a)-4(c) the measured contours of $\mathbf{E} \cdot \mathbf{B}$ are shown for Argon plasmas. The size of the diffusion region is independent of the ion mass. This conclusion is also supported by other measurements in Hydrogen, Helium, Nitrogen, and Argon plasmas (not shown). The number density of the plasma can be varied by changing the rf heating power. The size of the diffusion regions is also found to be independent of the number density (comparing the two last rows of Fig. 1). Hence, the size of the diffusion region does not scale with the electron or the ion inertial skin depths, $c / \omega_{e, i}$, and it is independent of the Larmor radii, $\rho_{i}$ and $\rho_{s}=\left(m_{i} T_{e}\right)^{1 / 2} /(q B)$. Furthermore, the shape of the current channel is different from the typical elongated Harris sheet observed in other experiments in the more collisional regimes [45], and its size largely exceeds the Larmor radius of the electrons, $\rho_{e}$. In [3] the high rates of reconnection observed in VTF were found consistent with a large neoclassical resistivity, caused by the absence of passing particles. Furthermore, in [5] it was found that within a distance from the $X$ line given by the electron drift orbit width, $\rho_{\text {cusp,e }}=\left(\rho_{e} l_{0}\right)^{1 / 2}$, the electrons do not follow the magnetic field lines. The electrons are therefore not effective in "short circuiting" the electric fields along B. In agreement with these predictions, Fig. 2(d) contains data that suggest that the width of the diffusion region scales with $\rho_{\text {cusp,e }}$

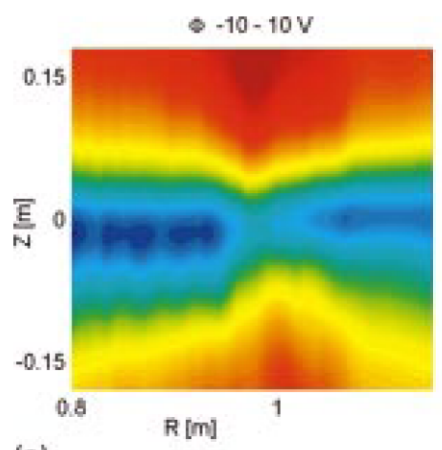

(a)

(d)

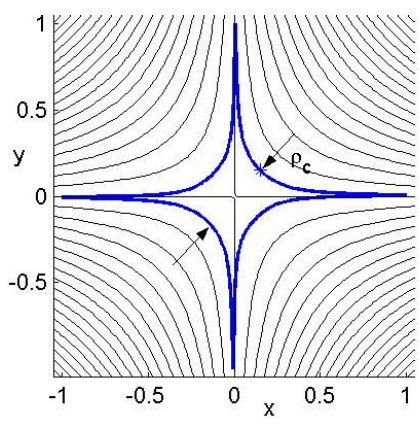

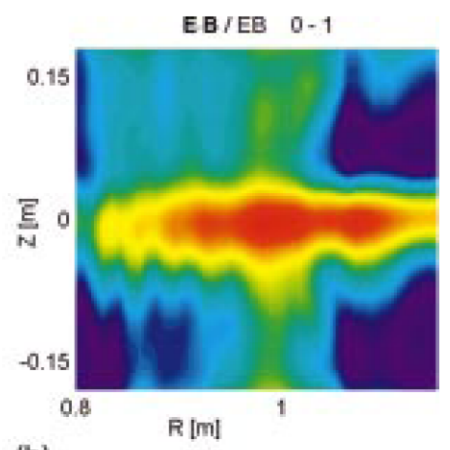

(b)

c)

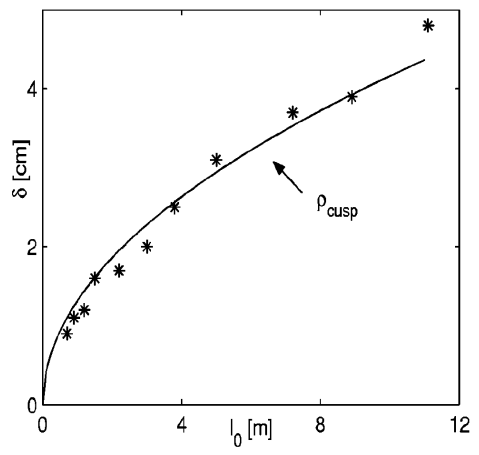

Figure 4: (a) Contours of electrostatic potential. (b) Calculated $E \cdot B$ based on the measured total electric field $\mathbf{E}$ $=-\nabla \Phi-\partial \mathbf{A} / \partial t$. The shape and size of the diffusion region is observed to be independent of the ion mass. (c) The width, $\delta$, of the diffusion region is observed to scale with the drift orbit width of the electrons, $\rho_{\text {cusp }, \mathrm{e}}=\left(\rho_{e} l_{0}\right)^{1 / 2}$.

\subsection{Oscillatory reconnection response}

To study the dynamic plasma behavior the identified oscillations were driven actively in Argon plasma by applying a reconnection drive oscillating at the observed resonance frequency. The details of the resonant plasma behavior are investigated by various measurements. We apply electrostatic and magnetic probe arrays for characterizing the poloidal profiles of key plasma parameters. The data in Fig. 5 were obtained in the time interval $60 \mu$ s to $132 \mu$ s covering a full 


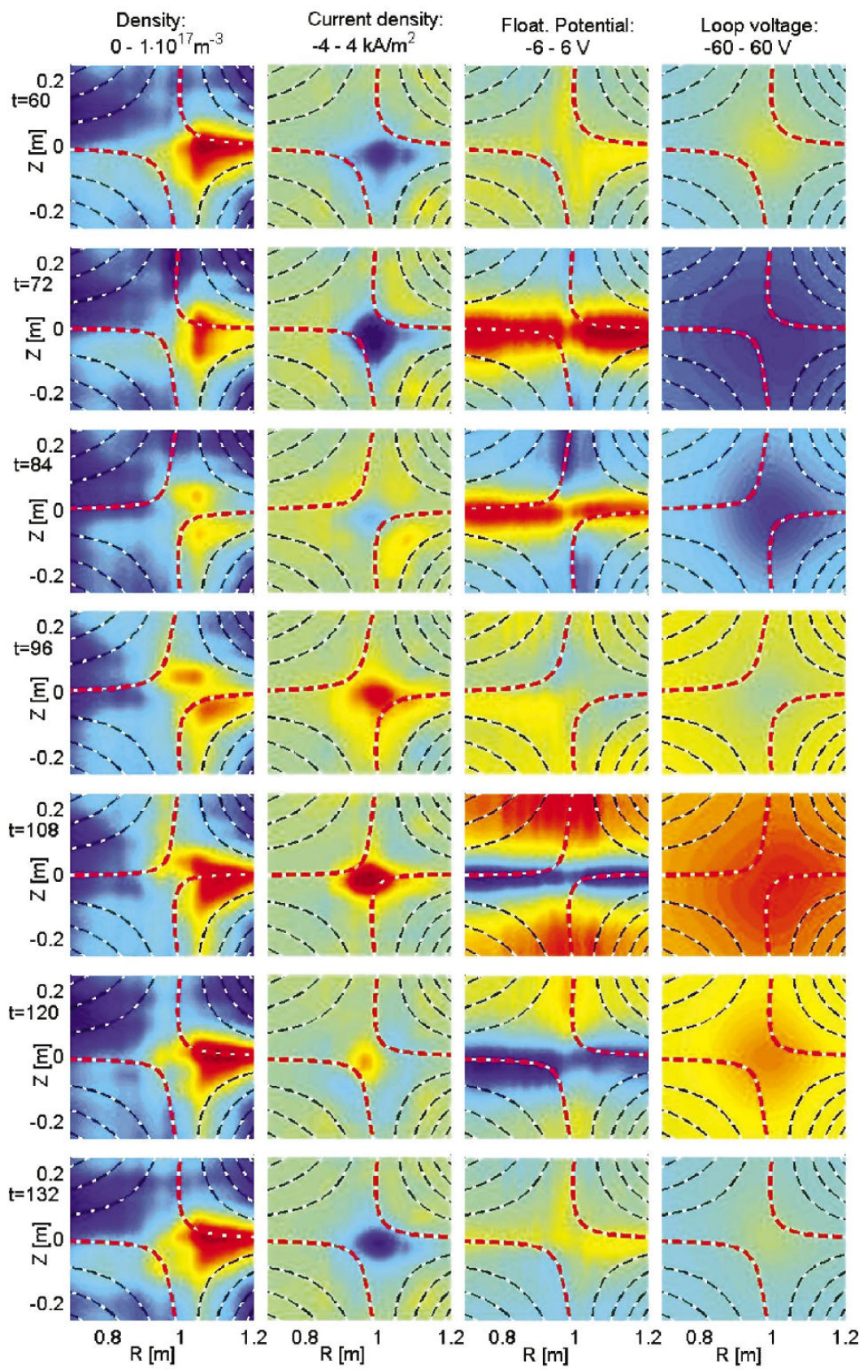

0

Figure 5: Measured contours of density, plasma current, floating potential, and loop voltage. Each row of subfigures corresponds to a snapshot in time as indicated on the left-hand side. Between each row the time is incremented by $12 \mathrm{~ms}$. The data are obtained during one full cycle of the reconnection drive. The data were obtained with $B_{\phi}=0.087 \mathrm{~T}, b_{0}=0.02 \mathrm{~T} / \mathrm{m}$, and thus $l_{0}=B_{\phi} / b_{0}=4.3 \mathrm{~m}$. 
period of the oscillatory reconnection drive. The poloidal magnetic flux function $\Psi$ was calculated on the basis of the currents applied in the VTF coil sets and the measured profiles of plasma current. Contours of $\Psi$ are shown in all the subfigures. The poloidal motion of the magnetic field lines is illustrated by the highlighted contours, which correspond to one particular value of $\Psi$. The first column of subfigures contains contours of plasma density, inferred from ion saturation current measurements. The modifications observed in the profiles are consistent with the plasma following the magnetic flux as it is reconnected back and forth across the separatrix.

The second column illustrates the measured contours of plasma current. The current densities are obtained from $\mathbf{j}=\nabla \times \mathbf{B} / \mu_{0}$, where $\mathbf{B}$ is measured by a magnetic probe array. In accordance with the oscillating reconnection drive we observe a periodic oscillation in the current profile. The last column shows the profiles of the loop voltage, $V_{\text {loop }}=-2 \pi d \Psi / d t$. The contours of the floating potential $\Phi$, shown in the third column, oscillate in phase with the loop voltage. The total electric field inside the plasma is given by $\mathbf{E}=-\nabla \Phi+\left(d V_{\text {loop }} / d t\right)(1 / 2 \pi R)$. In an ideal plasma the magnetic field and the plasma is frozen together such that $\mathbf{E}+\mathbf{v} \times \mathbf{B}=0$. For the case considered here with a finite guide magnetic field, this condition can only be fulfilled if $\mathbf{E} \cdot \mathbf{B}=0$. As discussed in Ref. 10 the role of the poloidal electric field $-\nabla \Phi$ is to ensure that $\mathbf{E} \cdot \mathbf{B}=0$ throughout the most of the cross section (the electron diffusion region can be identified as the region where $\mathbf{E} \cdot \mathbf{B}$ is finite). While the oscillations in the loop voltage $V_{\text {loop }}$ and the potential $\Phi$ are in phase, there is a noticeable phase delay between the plasma current and the loop voltage. The time behavior of the current profile is well described by a function of the form $j(R, Z, t)=V_{\text {loop }}(t) j_{1}$ $+d V_{\text {loop }}(t) / d t j_{2}$. Here we have introduced the time independent profiles $j_{1}(R, Z)$ and $j_{2}(R, Z)$ with units $\mathrm{AV}^{-1} \mathrm{~m}^{-2}$ and $\mathrm{AsV}^{-1} \mathrm{~m}^{-2}$, respectively. The profile, $\mathrm{j}_{1}$, is obtained from the time average of the measured current profile, $\mathrm{j}$, for an interval where $\left(d V_{\text {loop }} / d t\right)=0$.

As stated above, $\Phi$ and $V_{\text {loop }}$ oscillate in phase such that in the areas away from the separatrix the motion of the plasma is ideal $(\mathbf{E} \cdot \mathbf{B}=0)$. Thus, $\Phi$ can be written on the following form: $\Phi=V_{\text {loop }}(t) F(R, Z)$. Furthermore, the ion-polarization current, associated with this time varying electrostatic potential, can be described through the expression $\mathbf{j}_{\perp}=-d V_{\text {loop }} / d t\left(n m / B^{2}\right) \nabla_{\perp} F$. Given the spatial structure of $\Phi$ seen in Fig. 5 it follows that for $d V_{\text {loop }} / d t<0$ the ion-polarization current $j_{\perp}$ points away from the separatrix along $R-R_{0}=0$ and towards the separatrix along $Z=0$ (see Fig. 6). Field aligned electron currents $j_{\|}$flow to make closed "current loops" and maintain quasineutrality $[46,47]$.

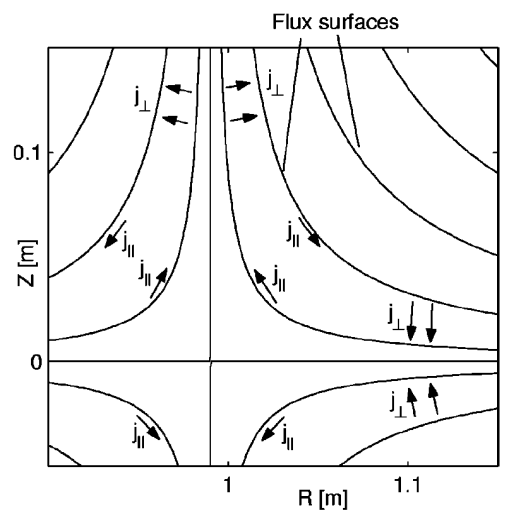

Figure 6: Illustration of the system of currents driven by time variations in $\Phi$. The ion-polarization currents $j_{\perp}$ and field-aligned electron currents form closed current loops. 


\subsection{Laser induced fluorescence measurements of the ions}

Observations in space and laboratory plasmas suggest magnetic reconnection as a mechanism for ion heating and formation of non-Maxwellian ion velocity distribution functions (IVDF). Below we present laser-induced fluorescence measurements of the IVDF parallel to the $X$ line of in the periodically driven experiments.

The intrinsically high spatial resolution of LIF allows one to perform local measurements of the IVDF. The laser is aligned to measure in the diffusion region around the $X$ line the ion velocity component $v_{\|}$parallel to the toroidal guiding magnetic field $B_{\phi}$. The size of the observation volume $(r \approx 1 \mathrm{~mm})$ is much smaller than the size of the diffusion region $(\delta \approx 4 \mathrm{~cm})$. The metastable argon II ion transition $3 d^{4} F_{7 / 2} \rightarrow 4 p^{4} D^{0}{ }_{5 / 2}$ at $\lambda=668.614 \mathrm{~nm}$ is pumped by a diode laser (60 $\mathrm{mW} \mathrm{cw}, 1 \mathrm{MHz}$ bandwidth). Via photomultiplier tube (PMT) and interference filter (1 $\mathrm{nm}$ bandwidth), the fluorescence signal of the transition $4 p^{4} D^{0}{ }_{5 / 2} \rightarrow 4 s^{4} P_{3 / 2}$ at $\lambda=442.72 \mathrm{~nm}$ is recorded.

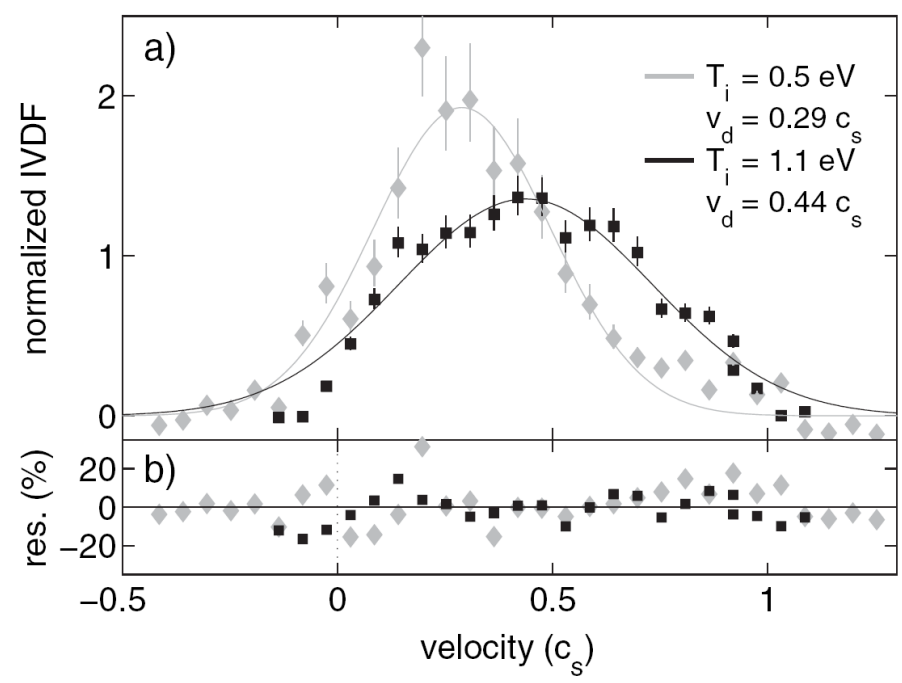

FIG. 7. (a) LIF measurements of the IVDF and the ion temperature. Error bars are obtained from the $95 \%$ confidence interval of the periodogram estimation. Gaussians are fitted to the measured data: without reconnection (gray diamonds) and with reconnection (black squares). (b) Residua (in \%) show the goodness of the two least-square fits.

Figure 7 summarizes the LIF measurement procedure for obtaining time-resolved IVDFs. The laser light is chopped "on/off" at a frequency $f_{d} / 2=7.5 \mathrm{kHz}$ and phase locked to the reconnection drive, which means that two reconnection periods are driven during each laser on/off cycle (see Fig. 1). The LIF signal is obtained by discriminating between the signals recorded with the laser on and laser off, respectively. For each laser wavelength $\lambda$, data from typically 30 reproducible plasma discharges are combined to obtain adequate signal to noise ratio. Tuning the laser over a suitable wavelength range yields the complete IVDF. Synchronizing chopper and drive allows for phase-locked averaging and thereby to resolve the fast time scale of the reconnection cycle (the time resolution of $\Delta t=5 \mu \mathrm{s}$ is restricted by the signal to noise ratio). Ion heating caused by reconnection was recently observed in the MRX device [48] and in the spheromak devices SSX [43] and TS-3 [38]. Ion energy balance calculations suggested that ion heating is caused by viscous heating and by nonclassical dissipation processes. In addition to the ion temperature increase, the mean drift velocity raises in VTF from $v_{d}=0.29 c_{s}$ to $v_{d}=0.44 c_{s}$, where $c_{s}=\left(T_{e} / m_{i}\right)^{1 / 2}$ is the ion sound speed. Toroidal ion drifts of the same order of magnitude were observed by Mach probes. 
Ion heating on the timescale of the reconnection drive was observed and is found to scale with the amplitude of the reconnection drive. In addition, nonthermal beam components were observed. Particle simulations reveal that ions passing the $X$-line region in the perpendicular direction are effectively heated by the in-plane electric field. Ion-ion collisions then lead to the observed parallel heating by a factor of $3-5$, localized at the $X$-line only. The in-plane electric fields are a general feature of reconnection with a strong guide magnetic field, emphasizing the importance of the present observations.

\subsection{Trapped electrons mediating fast reconnection}

The magnetic and electric fields throughout the poloidal cross-section are reconstructed experimentally during the reconnection process in VTF. The accurate characterization of these steady state profiles provides an excellent basis for detailed kinetic simulations of the reconnection process. With the known electric and magnetic fields Liouville's equation is readily solved numerically, providing the detailed phase space distribution function of the electrons. These distributions reveal non-Maxwellian features, which are fundamental in accounting for the momentum balance of the electrons in the vicinity of the $X$-line. Besides an introduction to the experimental geometry, here we describe these kinetic simulations of the reconnection process in VTF.

The mean free path between collisions for the electrons in VTF is in the order of $50 \mathrm{~m}$. The thermal speed of the electrons is typically $106 \mathrm{~m} / \mathrm{s}$, so during a particle confinement time, $\tau_{e}$ $\approx 0.2 \mathrm{~ms}$, the electrons execute hundreds of complete bounce orbits in the poloidal cross-sections. It is therefore natural to seek the explanation for the observed plasma behavior in the properties of the particle orbits; in Ref. [2] it was found that the width of the diffusion region scales with the drift orbit width of the electrons. An example of a characteristic electron orbit is in Fig. 8.a. A more detailed picture of the mechanisms responsible for the low current densities (and hereby fast reconnection) is obtained by solving Liouville's equations, $\mathrm{d} f / \mathrm{d} t=0$, for the electron phase space density. This equation states that the distribution is constant along particle orbits through phase space $(x, v)$. Hence, we can equate the distribution $f$, for a point $\left(\mathbf{x}_{0}, \mathbf{v}_{0}\right)$ in the reconnection region to an isotropic distribution, $f_{\infty}$, in the ambient. This is done by following particle orbits back in time until they reach points, $\left(\mathbf{x}_{1}, \mathbf{v}_{1}\right)$, in the ambient. It then follows that $f\left(\mathbf{x}_{0}, \mathbf{v}_{0}\right)=f_{\infty}\left(v_{1}\right), v_{1}=\left|\mathbf{v}_{1}\right|$.

The electron distributions are therefore closely related to changes in kinetic energy that the particles undergo along their trajectories into the X-line region. This scheme for obtaining the phase space distribution has been applied earlier in reconnection configurations with no guide magnetic field (no magnetic field component along the X-line) and no electrostatic in-plane potential [3]. However, due to the requirement of quasi neutrality, for the more generic reconnection configurations, in which a guide magnetic field is present, it is important also to include an in-plane electrostatic potential [4]. Because the structure of this potential is normally unknown the present numerical scheme had not been applied previously to configurations which do include a guide magnetic field. However, with our experimental knowledge of the electrostatic potential the scheme can be applied for the first time to configurations including a guide magnetic field. The particle orbits are solved by integrating the electron guiding center equations of motion in prescribed fields using a massively parallel computer code.

An example of the distribution function evaluated close to the X-line is shown in Fig. 8.b. As seen, the distribution contains strong non-Maxwellian features. Through associated terms in the electrons pressure tensor, these non-Maxwellian features are expected to be important for the momentum balance of the electrons throughout the reconnection region. The current profile is obtained from the first moment of the distribution function; an example is shown in Fig. 8.c. The numerical current density profiles are in good agreement with the experimental observations. Further investigations are underway to benchmark the numerical results against experimental data for a range of experimental parameters including, $E_{z}, l_{0}$ and $b_{0}$. 


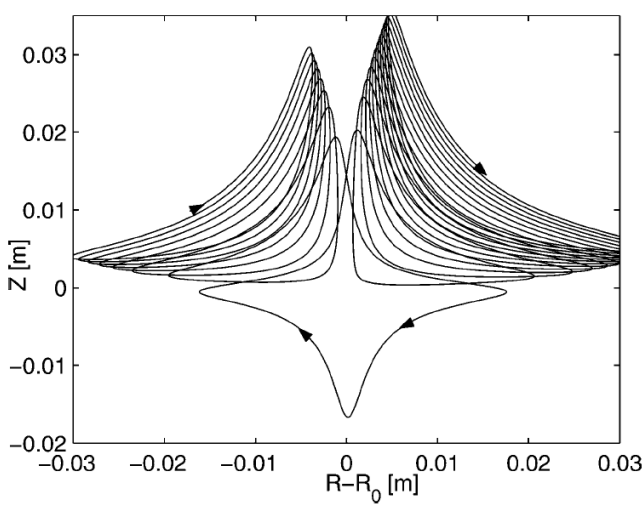

a)

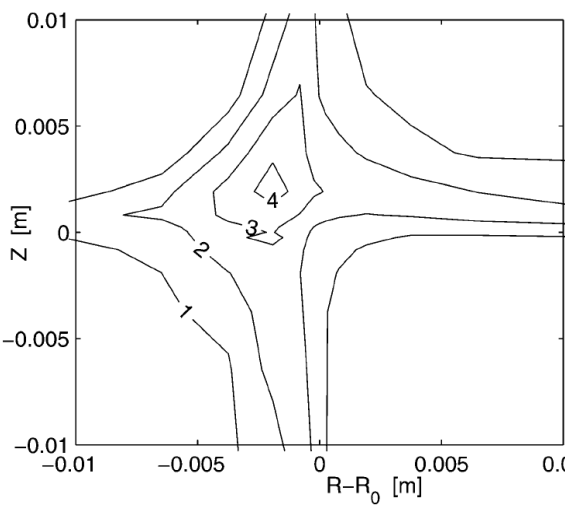

b)

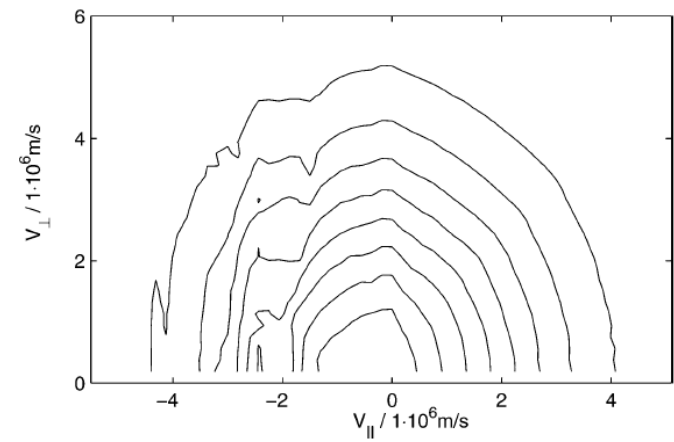

Figure 8: a) Example of an electron trajectory during reconnection in VTF. b) Contour of the electron velocity distribution for a location close to the X-line. c) Steady state current density calculated from the first moment of the electron velocity distribution. The contours levels (labeled in units, $\mathrm{kA} / \mathrm{m}^{2}$ ) are separated by $1 \mathrm{kA} / \mathrm{m}^{2}$.

\subsection{Application of the VTF open cusp results to space observations}

Recently, the first in situ measurements of the electron distribution function have been reported for a reconnection event encountered by the Wind spacecraft in Earth's magnetotail $[49,50]$. This event, observed in the deep magnetotail (60 Earth radii), is characterized by a kinetic regime in which the electron temperature is high, $T_{e} \approx 400 \mathrm{eV}$, and the magnetic field is weak, $B \approx 6-12 \mathrm{nT}$, implying an electron thermal speed, $v_{\text {th }}$, much larger than the Alfvén speed, $v_{A}$ (for the observed density $n \approx 0.1 \mathrm{~cm}^{-3}$ ), and thus larger than the outflow speed, $v_{\text {out }}$, of plasma from the reconnection region.

The reconnection dynamics of the VTF open configuration, largely influenced by the electrostatic field in the boundary layer between the plasma and the vessel wall, may at first appear unlikely to be relevant to reconnection in space. However, our analysis of Wind spacecraft observations of reconnection has documented a similar behavior of trapped electrons in the deep magnetotail $\left(>50 R_{E}\right.$ ). For the reconnection event encountered by Wind the entire diffusion region is positively charged. This potential effectively traps all thermal electrons (just as is the case with the electric fields at the vacuum vessel walls in VTF).

An example of electron distribution measured as Wind passed through the ion diffusion region is displayed in Fig. 9 right. The measurements provide the phase-space density, $f$, of the electrons as a function of pitch angle, $\angle(\mathbf{v}, \mathbf{B})$, and kinetic energy, $E_{k}$. For the reconnection event encountered by Wind the entire diffusion region is positively charged. This potential effectively traps all thermal electrons (just as is the case with the electric fields at the vacuum vessel walls in VTF). The data in Fig. 9 left, recently published in Physics Review Letters [9], illustrate how the VTF kinetic model for reconnection reproduces the details of the electron distribution function measured by Wind. 
Perhaps the most important effect of this trapping is that it implies a nearly symmetric bounce motion of the electrons along the magnetic field; a symmetry which is also reflected in their distributions function $f\left(v_{\|}, v_{\perp}\right) \approx f\left(-v_{\|}, v_{\perp}\right)$. For reconnection geometries including a guide magnetic field it follows that the current along the reconnection X-line must be limited, $j_{\|}=\int_{v_{\|} f d^{3} v}$ $\approx 0$. Thus, the kinetic behavior of the trapped electrons causes a locally enhanced resistivity, which in a fluid description would appear as a finite $\nabla \cdot \mathbf{P}$-term in the generalized Ohm's law.
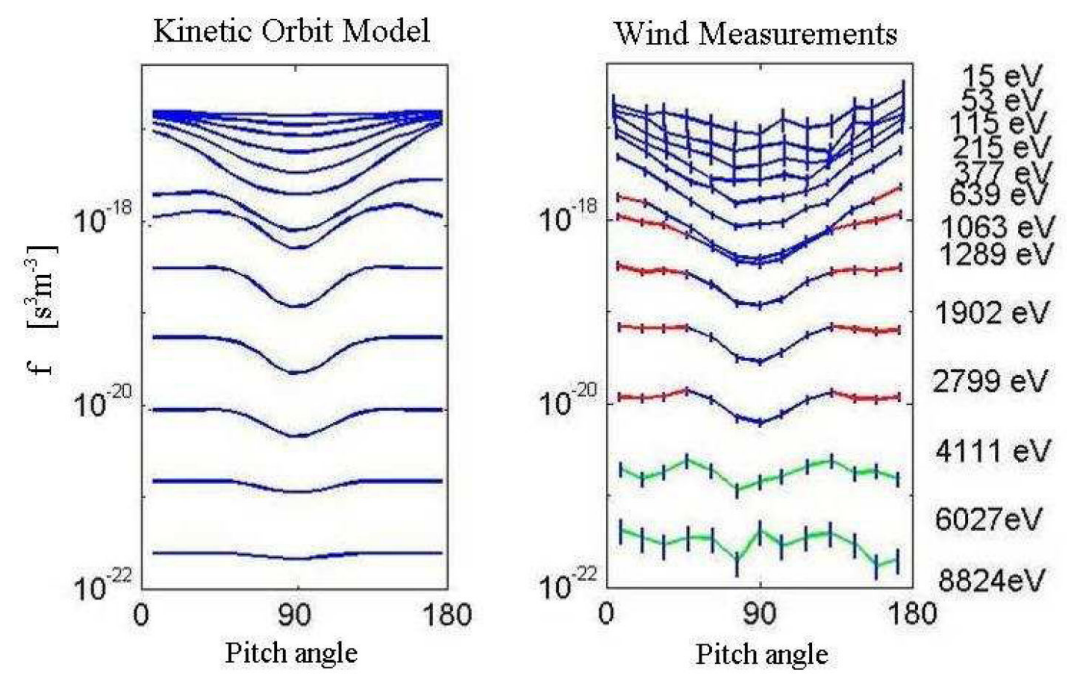

Figure 9: Right: Electron phase-space distribution measured in situ by the WIND spacecraft in the Earth's magnetotail in the center of an active reconnection region. Left: The details of the anisotropy (the pitch angle dependence) in the WIND measurements are reproduced by the kinetic model for reconnection developed on the basis of the VTF experimental observations.

\subsection{Spontaneous reconnection in the new closed VTF configuration}

The focus of our most resent investigations is reconnection in a closed configuration realized in VTF by the installation of in-vessel coils. Figure 10(a) provides a view inside the VTF vacuum vessel during the installation of these coils implementing the closed magnetic configuration. As is explained in a later section the configuration includes four coils (rather than just two) to actively trigger reconnection. The coils are suspended in four locations by thin stainless steel wires. In the initial phase of the experiment the current is induced with an identical value and direction in all four coils. This produces the configuration illustrated in Fig. 10(b), which provides excellent plasma confinement. At the midplane of the configuration, $Z=0$, a magnetic X-line is formed, easily accessible with magnetic and electrostatic probes. The closed configuration includes toroidally passing electron orbits and as a result the total plasma current is increased by three orders of magnitude as compared to the currents measured in the open configuration. 


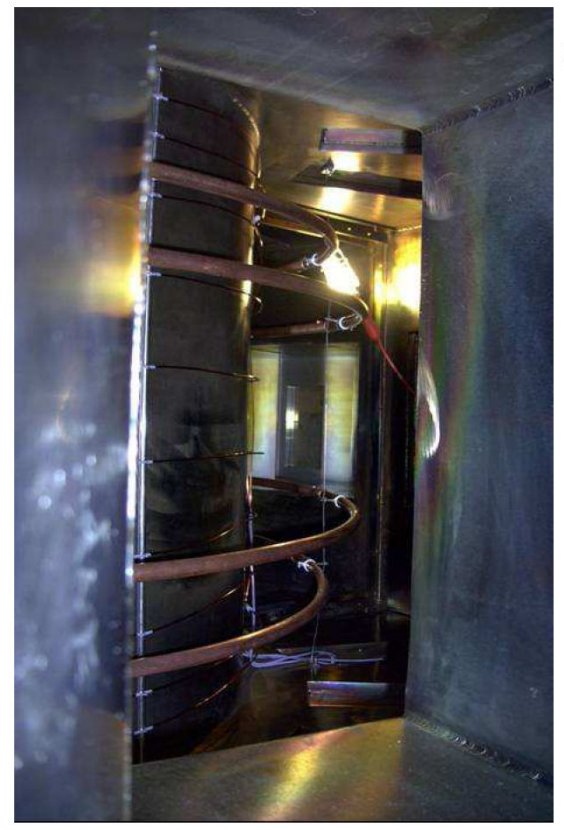

(a)

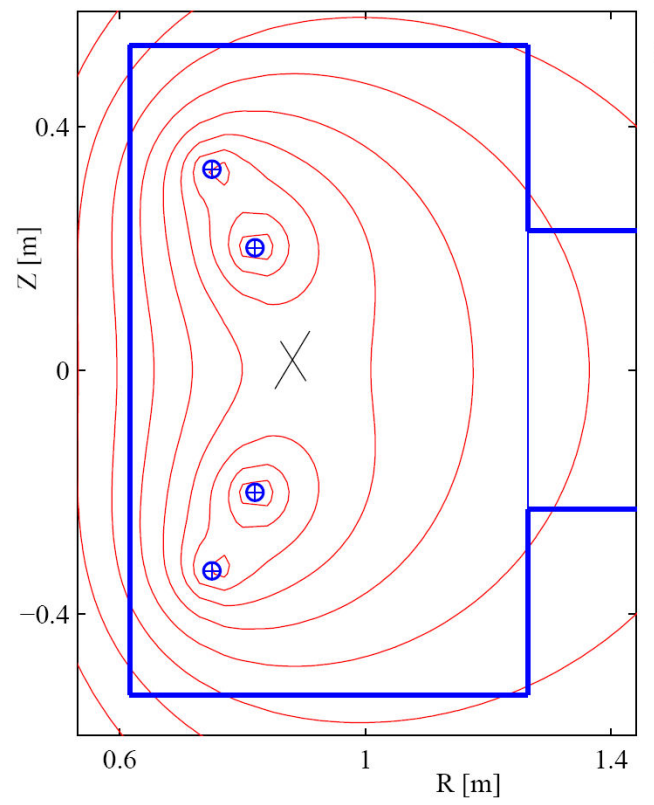

(b)

Figure 10: (a): View in side the VTF vacuum vessel during the installation of the four co-axial loops implementing the closed magnetic configuration. (b): Cross section of the VTF devices including contours of constant poloidal magnetic flux as produced by the four internal coils (note that the poloidal projection of the magnetic field lines coincides with the contours of constant flux).

\begin{tabular}{|c|c|c|c|c|c|}
\hline Parameter & MRX & LCD & TS-D3 & $\begin{array}{l}\text { VTF } \\
\text { open }\end{array}$ & $\begin{array}{l}\text { VTF } \\
\text { closed }\end{array}$ \\
\hline [m] & 0.1 & 0.3 & 0.1 & 0.5 & 0.2 \\
\hline$n_{e}\left[\mathrm{~m}^{-3}\right]$ & $10^{19}-10^{20}$ & $10^{18}$ & $5 \cdot 10^{20}$ & $2 \cdot 10^{17}$ & $10^{17}-10^{19}$ \\
\hline$T_{e} \quad[\mathrm{eV}]$ & 20 & 10 & 10 & 20 & -60 \\
\hline$T_{i} \quad[\mathrm{eV}]$ & 10 & 2 & 10 & 2 & $-\quad 15$ \\
\hline$[\mathrm{T}]$ & 0.05 & $2 \cdot 10^{-3}$ & 0.5 & $0.03-0.09$ & $0.03-0.09$ \\
\hline$m_{i} / m_{p}$ & 1 & 40 & 4 & -40 & -40 \\
\hline$\rho_{i} / L$ & 0.08 & 2 & 0.01 & $0.03-0.09$ & $0.02-0.6$ \\
\hline$c / \omega_{p i}[\mathrm{~m}]$ & 0.02 & 0.6 & $2 \cdot 10^{-3}$ & $0.5-3$ & $0.07-$ \\
\hline$c / \omega_{p e}[\mathrm{~m}]$ & $5 \cdot 10^{-4}$ & $5 \cdot 10^{-}$ & $2 \cdot 10^{-4}$ & 0.01 & $0.002-0.02$ \\
\hline$[\mathrm{m}]$ & $0.002-($ & 1 & $1 \cdot 10^{-3}$ & $10-100$ & $0.1-10^{3}$ \\
\hline$S$ & 900 & 60 & 700 & 900 & $150-4500$ \\
\hline
\end{tabular}

Table 1: Plasma parameters characterizing a selection of reconnection experiments. The length $\mathrm{L}$ is the smallest dimension of the chamber. ne and Te are the electron density and temperature, respectively, $\mathrm{Ti}$ is the ion temperature, $\mathrm{B}$ is the magnetic field strength, $\mathrm{mi} / \mathrm{mp}$ is the ion mass compared to the proton mass, $\rho \mathrm{i}$ is the ion Larmor radius, $\rho$ is the electron mean free path, and $\mathrm{S}$ is the Lundquist number. 
Our preliminary investigations have shown that the in-vessel coils enable the collisionallity to be effectively controlled by the neutral gas pressure. Experimentally it has been verified that the ranges $\mathrm{n}_{\mathrm{e}} \approx 10^{17} \mathrm{~m}^{-3}-10^{19} \mathrm{~m}^{-3}$ and $\mathrm{T}_{\mathrm{e}} \approx 60-5 \mathrm{eV}$ are accessible; this corresponds to a range in the electron mean free path that covers four orders of magnitude. At the highest electron temperatures the Lundquist number approaches $S \approx 10^{4}$ which makes the experiment relevant to reconnection in magnetic fusion experiments. Note that, due to the magnetic field of the solid conductors, the current channel is stable with respect to the hoop force.

The plasma dynamics are reconstructed from data acquired in a single discharge by electrostatic and magnetic probes. A 196 channel electrostatic probe array provides information on the electrostatic fields and the plasma density for the entire poloidal cross section; the floating potential of the plasma is measured at 98 locations simultaneous with 98 ion-saturation currents measurements. In addition, a novel flux-probe array is used to measure $\partial \Psi / \partial t$ directly: 98 pickup loops extend across the poloidal cross section. Their widths are proportional to $R$, and they are arranged to measure

$$
\Delta \dot{\Psi}(R, Z)=\dot{\Psi}(R, Z)-\dot{\Psi}\left(R_{0}, Z_{0}\right)=\int_{R_{0}, Z_{0}}^{R, Z} R^{\prime} \mathbf{B} \times d \mathbf{l} \text { on a grid covering the central }
$$

reconnection region. At the location $\left(R_{0}, Z_{0}\right)$ (at the inner vessel wall) $\dot{\Psi}\left(R_{0}, Z_{0}\right)$ is measured by a toroidal loop, completing the measurement of $\dot{\Psi}(R, Z)$. At a separate toroidal angle $\Delta \dot{\Psi}(\mathrm{R}, \mathrm{Z}=0)$ is measured. The measurements of the reconnection rate at the two toroidal location $\left(180^{\circ}\right.$ apart) prove that the reconnection is mostly axisymmetric. Using Maxwell's equations, the poloidal magnetic fields and the toroidal plasma current density are computed from $\Psi=\int \dot{\Psi} d t$. Figure 11 shows the measured contours of the plasma density, electrostatic floating potential, current density, and reconnection rate during a $65 \mu$ s time interval. Note that the time intervals between the columns are nonuniform. The dashed magnetic field lines are obtained as the contours of constant $\Psi$ (directly measured). In the sequence for $t \leq 75 \mu$ s the reconnection rate is on the order of $2 \mathrm{~V} / \mathrm{m}$. In this period the broad current profile is intensified and acquires a significant elongation; the width of the current sheet is reduced to about $0.1 \mathrm{~m}$, while the magnetic geometry becomes increasingly stretched.

The profiles at $t=80 \mu \mathrm{s}$ mark the onset of the reconnection event. The value of $E_{\text {rec }}=-$ $(\partial \Psi / \partial t) / R$ jumps to $14 \mathrm{~V} / \mathrm{m}$ at the upper outflow region of the current sheet. Hereafter the elevated value of $-(\partial \Psi / \partial t) / R$ engulfs the entire reconnection region as the current channel decays away. The highlighted field lines can be followed in time as they move into the $X$ line, reconnect, and drift apart. The plasma density contours show how the central density is ejected downwards at a velocity consistent with the motion of the highlighted magnetic field lines. The outflow velocity is about $v_{\text {out }} \approx 11 \mathrm{~km} / \mathrm{s}$, corresponding to an energization of $\mathrm{E}_{\text {flow }} \approx 24 \mathrm{eV}$. 

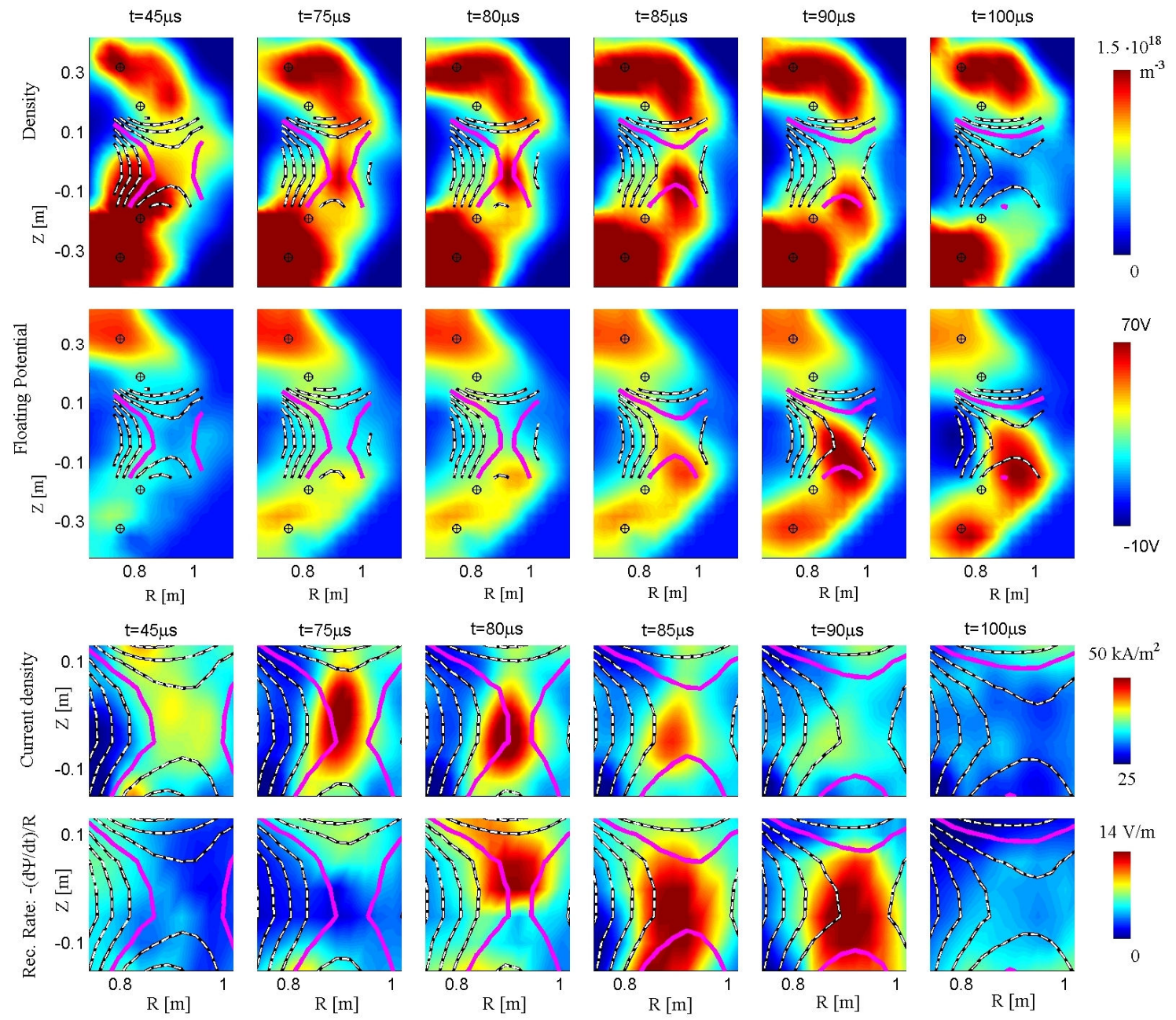

Figure 11: Measured contours of the plasma density, floating potential, current density, and the reconnection rate. The overlaid lines represent contours of $\Psi$, which coincide with the poloidal projection of magnetic field lines. One value of $\Psi$ is highlighted such that the motion of the field lines can be followed in time. The measurements were obtained in a $65 \mu$ s time interval centered about a spontaneous reconnection event. Note that the time steps between the columns are non-uniform.

The period of slow reconnection after the tearing drive is applied allows the magnetic stress to accumulate in the system. During this phase a current channel is formed which becomes increasingly intense and elongated. A sudden onset of reconnection is observed as the width of the current sheet approaches the ion-sound-Larmor radius $\rho_{s}$. During the event the plasma in the reconnection region is ejected. Significant electron heating is observed and the evolution of $\Phi$ indicates that the ions are accelerated to the $\mathbf{E} \times \mathbf{B}$ velocity (out of the region) by $\mathbf{j} \times \mathbf{B}$ forces. The spontaneous reconnection events occur only for a limited range of the plasma parameters available in VTF. Our future studies will map the parameter regimes yielding the spontaneous events. Also, we will study possible 3D effects at the onset of the reconnection events. 


\section{Outlook: Three dimensional effects in the onset of fast reconnection}

The continued operation of the facility has been secured by a DOE junior faculty award for Jan Egedal, who recently was promoted to Assistant Professor in the MIT Physics department. Profs. Egedal and Porkolab have also been awarded an NSF/DOE award to continue the studies initiated over the past funding period. At presence, the main focus of the experiment is the three dimensional plasma dynamics observed in the onset of fast reconnection.

References (paper published under this grant are in bold)

[1] J. Egedal, A. Fasoli, M. Porkolab and D. Tarkowski, "Plasma generation and confinement in a toroidal magnetic cusp", Rev. Sci. Instrum. 71, 3351, (2000).

[2] J. Egedal, A. Fasoli, D. Tarkowski and A. Scarabosio, "Collisionless magnetic reconnection in a toroidal cusp", Physics of Plasma, 8, 1935, (2001).

[3] J. Egedal and A. Fasoli, "Single-particle dynamics in collisionless magnetic reconnection", Phys. Rev. Lett., 86, 5047, (2001).

[4] J. Egedal and A. Fasoli, "The topology of guiding center orbits in a linear magnetic cusp", Physics of Plasmas, 8. 4042, (2001).

[5] J. Egedal, "A drift kinetic approach to reconnection in an open cusp plasma", Physics of Plasmas, 9, 1095, (2002).

[6] J. Egedal, A. Fasoli and J. Nazemi, "Dynamical Plasma Response during Driven Magnetic Reconnection", Phys. Rev. Lett. 90, 135003, (2003).

[7] J. Egedal, W. Fox, M. Porkolab and A. Fasoli, "Experimental evidence of fast reconnection via trapped electron motion", Physics of Plasmas, 11, 2844, (2004).

[8] J. Egedal, W. Fox, E. Bolonohy and M. Porkolab, "Kinetic simulation of the VTF magnetic reconnection experiment", Computer Physics Communications, 164, 29, (2004).

[9] J. Egedal, M. Oieroset, W. Fox and RP. Lin, "In situ discovery of an electrostatic potential, trapping electrons and mediating fast reconnection in the Earth's magnetotail", Phys. Rev. Lett. 94, 025006, (2005).

[10] J. Egedal, W. Fox, M. Porkolab and A. Fasoli, "Eigenmode response to driven magnetic reconnection in a collisionless plasma", Physics of Plasmas, 12, 052107, (2005).

[11] A. Stark, W. Fox, J. Egedal, et al., "Laser-induced fluorescence measurement of the ion-energy-distribution function in a collisionless reconnection experiment ", Phys. Rev. Lett. 95, 235005, (2005).

[12] J. Egedal, W. Fox, N. Katz, M. Porkolab, K. Reim, E. Zhang, "Laboratory Observation of Spontaneous Magnetic Reconnection", Phys. Rev. Lett. 98, 015003, (2007).

[13] J. W. Dungey, Phil. Mag. 44, 725 (1953).

[14] J. B. Taylor, Rev. Mod. Phys. 28, 243 (1986).

[15] V. M. Vasyliunas, Rev. Geophys. Space Phys. 13, 303 (1975).

[16] T. D. Phan, et. al Nature 404, no.6780, pp.848-50 (2000).

[17] S. Masuda, T. Kosugi, H. Hara and Y. Ogawara, Nature 371, 495 (1994).

[18] R. M. Kulsrud, Phys. Plasmas 2, 1735 (1995).

[19] P. A. Sweet, Nuovo Cimento Suppl. 8, Ser. X, 188 (1958).

[20] E. N. Parker, J. Geophys. Res. 62, 509 (1957).

[21] B. Coppi and A. B. Friedland, Astrophys. J. 29, 379 (1971).

[22] J. Heyvaerts, E. Priest, and D. Rust, Astrophys. J. 216, 123 (1977).

[23] J. Birn, J. F. Drake, M. A. Shay, et al., J. Geophys. Res. 106 (A3), 3715 (2001). 
[24] M. A. Shay, J. F. Drake, B. N. Rogers and R. E. Denton, Geophys. Res. Lett. 26, 2163 (1999).

[25] A. Aydemir, Phys. Fluids B 4, 3469 (1992).

[26] R. Kleva, J. Drake, and F. Waelbroeck. Phys. Plasmas 2, 23 (1995).

[27] B. N. Rogers, R. E. Denton, J. F. Drake, and M. A. Shay, Phys. Rev. Lett. 87, 195004 (2001).

[28] J. F. Drake, M.A. Shay, W. Thongthai, et al., Phys. Rev. Lett. 94, 095001 (2005).

[29] P. L. Pritchett, Phys. Plasmas 12, 062301 (2005).

[30] J. A. Wesson, Nuclear Fusion 30, 2545 (1990).

[31] F. Porcelli, D, Borgogno, F. Califano, et al., 44, 389 (2002).

[32] P. L. Pritchett, J. Geophys. Res. 106 (A11), 25961 (2001).

[33] D. Overskei and P. A. Politzer, Phys. Fluids 19, 683 (1976).

[34] G. G. Zukakishvili, I. F. Kavartskhava and L. M. Zukakishvili, Sov. J. Plasma Phys. 4, 405 (1978).

[35] R. L. Stenzel and W. Gekelman, J. Geophys. Res. 86, 649 (1981).

[36] A. T. Altyntsev, V. M. Bardakow, V. I. Krasov, N. V. Lebedev and V. L, Paperni, Solar Phys. 106, 131 (1986).

[37] S. V. Bulanov and A. G. Frank, Sov. J. Plasma Phys. 18, 797 (1992).

[38] Y. Ono, M. Yamada, T. Akao, T. Tajima and R. Matsumoto, Phys. Rev. Lett. 76, 3328 (1996).

[39] M. Yamada, H. T. Ji, S. Hsu, T. Carter, R. Kulsrud, N. Bretz, F. Jobes, Y. Ono and F. Perkins, Phys. Plasmas 4, 1937 (1997).

[40] M. R. Brown, Phys. Plasmas 6, 1717 (1999).

[41] Y. Ono, M. Inomoto, T. Okazaki and T. Ueda, Phys. Plasmas 4, 1953 (1997).

[42] T. W. Kornack, P. K. Sollins and M. R. Brown, Phys. Rev. E 58, R36 (1998).

[43] M. R. Brown, C. D. Cothran, M. Landreman, D. Schlossberg, W. H. Matthaeus, G. Qin, V. S. Lukin, and T. Gray, Phys. Plasmas, 9, 2077 (2002).

[44] T. A. Carter, H. Ji, F. Trintchouk, M. Yamada, and R. M. Kulsrud, Phys. Rev. Lett. 88, $015001(2002)$

[45] H. Ji, M. Yamada, S. Hsu, and R. Kulsrud, Phys. Rev. Lett. 80, 3256 (1998).

[46] A. Aydemir, Phys. Fluids B 4, 3469 (1992).

[47] R. Kleva, J. Drake, and F. Waelbroeck, Phys. Plasmas 2, 23 (1995).

[48] S. C. Hsu et al., Phys. Rev. Lett. 84, 3859 (2000).

[49] M. Øieroset et al., Nature (London) 412, 414 (2001).

[50] M. Øieroset et al., Phys. Rev. Lett. 89, 195001 (2002). 